\title{
Early identification of patients at risk for acute respiratory distress syndrome among severe pneumonia: a retrospective cohort study
}

\author{
Jian Luo ${ }^{1 *}$, He Yu ${ }^{2 *}$, Yue-Hong Hu ${ }^{1}$, Dan Liu ${ }^{2}$, Yi-Wei Wang ${ }^{1}$, Mao-Yun Wang ${ }^{1}$, Bin-Miao Liang ${ }^{1}$, Zong- \\ An Liang ${ }^{1}$
}

${ }^{1}$ Department of Respiratory Diseases, ${ }^{2}$ Department of Critical Care Medicine, West China School of Medicine and West China Hospital, Sichuan University, Chengdu 610041, China

Contributions: (I) Conception and design: J Luo, H Yu, BM Liang, ZA Liang; (II) Administrative support: BM Liang, ZA Liang; (III) Provision of study materials or patients: H Yu, ZA Liang; (IV) Collection and assembly of data: J Luo, YH Hu, D Liu; (V) Data analysis and interpretation: J Luo, H Yu, YW Wang, MY Wang; (VI) Manuscript writing: All authors; (VII) Final approval of manuscript: All authors.

*These authors contributed equally to this work.

Correspondence to: Bin-Miao Liang; Zong-An Liang. Department of Respiratory Diseases, West China School of Medicine and West China Hospital, Sichuan University, No.37, Guoxue Alley, Chengdu 610041, China. Email: liangbm0202@yahoo.com; liangza0816@yahoo.com.

Background: Severe pneumonia is the predominant cause for acute respiratory distress syndrome (ARDS). Identification of ARDS from patients with severe pneumonia remains a significant clinical problem due to the overlap of clinical presentations and symptoms. Early recognition of risks for ARDS from severe pneumonia is of great clinical value.

Methods: From April 2014 to December 2015, patients with severe pneumonia at admission were retrieved from the hospital database, of which ARDS developed within 7 days were further identified. We compared the demographic and clinical characteristics at admission between severe pneumonia patients with and without ARDS development, followed by analysis of potential predictors for ARDS development and mortality. Multivariate logistic regression and receiver operating characteristic (ROC) curves were performed to screen independent risk factors and identify their sensitivity in predicting ARDS development and prognosis.

Results: Compared with severe pneumonia without ARDS development, patients with ARDS development had shorter disease duration before admission, higher lung injury score (LIS), serum fibrinogen (FiB), and positive end-expiratory pressure (PEEP), lower Marshall score, sequential organ failure assessment score and proportion of cardiovascular and gastrointestinal diseases, but similar mortality. Serum FiB $>5.15 \mathrm{~g} / \mathrm{L}$ [adjusted odds ratio (OR) 1.893, 95\% confidence interval (CI): 1.141-3.142, $\mathrm{P}=0.014$ ] and $\mathrm{PEEP}>6.5 \mathrm{cmH}_{2} \mathrm{O}$ (adjusted OR 1.651, 95\% CI: 1.218-2.237, P=0.001) were independent predictors for ARDS development with a sensitivity of $58.3 \%$ and $87.5 \%$, respectively, and $\mathrm{pH}<7.35$ (adjusted OR 0.832, 95\% CI: 0.702-0.985, $\mathrm{P}=0.033$ ) was an independent risk factor for ARDS mortality with a sensitivity of $95.2 \%$.

Conclusions: ARDS development risk could be early recognized by PEEP $>6.5 \mathrm{cmH}_{2} \mathrm{O}$ and serum $\mathrm{FiB}>5.15 \mathrm{~g} / \mathrm{L}$ in severe pneumonia patients, and $\mathrm{pH}<7.35$ is a reliable prognostic factor in predicting ARDS mortality risk.

Keywords: Acute respiratory distress syndrome (ARDS); severe pneumonia; risk factors; fibrinogen (FiB); positive end-expiratory pressure (PEEP); $\mathrm{pH}$

Submitted Mar 09, 2017. Accepted for publication Aug 29, 2017.

doi: $10.21037 /$ jtd.2017.09.20

View this article at: http://dx.doi.org/10.21037/jtd.2017.09.20 


\section{Introduction}

Acute respiratory distress syndrome (ARDS) is a type of acute diffuse and inflammatory lung injury with an estimated mortality of approximately $40 \%(1-5)$. It is a form of pulmonary edema without cardiogenic origin and can be induced by either pulmonary or systemic inflammatory process such as pneumonia and severe acute pancreatitis. The clinical landmarks of ARDS are severe refractory hypoxemia and acutely processing in disease course, which usually results in difficulty and failure in the treatment of patients with ARDS status $(6,7)$. Therefore, early recognition of patients with high risk for developing ARDS and the subsequent prevention of its progress is of great value in critical care units $(8,9)$.

Severe pneumonia has been reported as the predominant cause in pulmonary originated ARDS, and identification and diagnosis of ARDS from patients with severe pneumonia remains a significant clinical problem due to the overlap of clinical presentations and symptoms in both diseases $(10,11)$. Although studies have demonstrated numerous risk factors for the development of ARDS including aspiration, inappropriate mechanical ventilation (MV), extrapulmonary sepsis, polytrauma, and pancreatitis $(1,12)$, and the prognosis of ARDS such as acute cor pulmonale, Cytomegalovirus seroprevalence, shock on hospital admission, and acute physiology, age, chronic health evaluation II (APACHE II) score (13-15), but most of these risk factors are also true in patients with severe pneumonia. Therefore, identification of risk of ARDS development in patients with severe pneumonia seems more challenging and more practical to clinicians.

Recently, an acute lung injury (ALI) prediction model, the Lung Injury Prediction Score (LIPS), was introduced and validated to discriminate patients at high risk of ALI before intensive care unit admission $(8,16)$. It integrates the risk factors and modifiers present at the time of hospital admission with the aim of determining population at high risk before ALI onset. In spite of the potential power of early identification, the underlying diseases and causes for ALI and ARDS were diverse, and specific factors should be considered in an individual disease, therefore, integrated assessment might contribute to potential underestimation in certain disease conditions. In postoperative patients, Kim et al. and Chen $e t$ al. separately reported risk factors for ARDS development in patients underwent pneumonectomy and valvular heart surgery, and they identified that low predicted postoperative forced expiratory volume in 1 second, high perfusion fraction of resected lung, old age, presence of liver cirrhosis, massive blood transfusion, and tricuspid valve replacement resulted in higher prevalence of ARDS $(17,18)$. Nevertheless, relevant studies in specific patients with severe pneumonia are scarce, which intrigued and drove us investigating potential risk factors for patients with severe pneumonia progressed to ARDS.

Based on the ambiguous perceptions and heterogeneous patients investigated, we hypothesized that individual risk factors should be associated with ARDS development in patients with severe pneumonia; therefore a retrospective case-control study was conducted to further identify risk factors associated with ARDS development and prognosis in order to early recognize ARDS from severe pneumonia and determine the optimal treatment strategies.

\section{Methods}

From April 2014 to December 2015, patients were screened for eligibility once they were admitted to respiratory and infectious intensive care unit (RICU) of West China Hospital, Sichuan University. Patient information was retrieved from our previous randomized control trial (10), and the study protocol was approved by the Institutional Ethical Committee for Clinical and Biomedical Research of West China Hospital [No. ChiCTR-TRC-14004163; Universal Trial Number (UTN): U1111-1152-2390]. Written informed consent was obtained from the legal substitute decision makers or lineal consanguinity for each patient. All methods were performed in accordance with the relevant guidelines and regulations released by the Chinese National Institutes of Health and the Clinical Trial Center of West China Hospital.

\section{Study design}

Two individual steps of comparison and analysis were performed in our study, in which eligible patients were classified into severe pneumonia patients who developed ARDS and those who did not, and patients with ARDS development were further divided into survival and dead groups. In step 1, we compared the demographic, clinical and prognostic characteristics on admission between patients with and without ARDS development to screen the potential indicators for ARDS development. In step 2, we divided patients with ARDS development into survival and death to analyze the related risk factors for ARDS mortality. 


\section{Participants}

Patients with severe pneumonia on admission were included in our retrospective analysis based on the consensual criteria released by Infectious Diseases Society of America and American Thoracic Society, which met either major criteria (acute respiratory failure requiring invasive $\mathrm{MV}$ and septic shock with need for vasopressors) or at least three minor criteria (respiratory rate $\geq 30 \mathrm{bpm}$, ratio of partial pressure of arterial oxygen to fraction of inspired oxygen $\left(\mathrm{PaO}_{2} / \mathrm{FiO}_{2}\right)$ $\leq 250$, blood urea nitrogen (BUN) $\geq 20 \mathrm{mg} / \mathrm{dL}$, white blood cell count $<0.4 \times 10^{9} / \mathrm{L}$, platelet count $<100 \times 10^{9} / \mathrm{L}$, body temperature $<36{ }^{\circ} \mathrm{C}$, multilobar infiltrates, confusion/ disorientation, and hypotension requiring aggressive fluid resuscitation) (19). Severe pneumonia patients with ARDS development were defined as ARDS occurring within 7 days after admission according to the 1994 AmericanEuropean Consensus Conference Definition: (I) acute onset of respiratory symptoms; (II) bilateral infiltrates in chest radiograph; (III) left atrial hypertension and cardiogenic pulmonary edema ruled out by echocardiography; (IV) severe hypoxemia with $\mathrm{PaO}_{2} / \mathrm{FiO}_{2} \leq 300 \mathrm{mmHg}$ (2).

We excluded patients if they had alternative pulmonary diseases regardless of the presence of pneumonia such as acute exacerbation of chronic obstructive pulmonary disease, asthma, and pulmonary thromboembolism, or underwent fatal and life-threatening comorbidities including but not limited to aggressive carcinoma with unstable clinical conditions, and cerebral injury. Patients with immunosuppressive therapy were also excluded in our study.

\section{Treatment strategies}

Pathogens identification was conducted upon the patients' admission by blood, sputum, or urine culture for bacteria and fungi, or chemiluminescence for virus specific IgM in serum, and empirical and subsequent pathogen-directed antimicrobial therapies were administered according to the consensus guidelines (19). For mechanical ventilation, "lung protective ventilation" and "open-lung approach" were performed, and we used volume-controlled mode to target the tidal volume of $6 \mathrm{~mL} / \mathrm{kg}$ of predicted body weight with allowances for 4 to $8 \mathrm{~mL} / \mathrm{kg}$ and the plateau airway pressures of not exceeding $30 \mathrm{cmH}_{2} \mathrm{O}$. Positive endexpiratory pressure (PEEP) was adjusted by $\mathrm{FiO}_{2}$ to meet the $\mathrm{PaO}_{2} \geq 55 \mathrm{mmHg}$ or oxygen saturation of pulse oximetry $\left(\mathrm{SpO}_{2}\right) \geq 88 \%(3,20)$. On the basis of analgesia by fentanyl, we sedated the patients with midazolam and/or propofol to meet the goal of Richmond Agitation-Sedation Scale between -2 and -4 (21). Additional treatment strategies such as organ function support, circulation stabilization, nutrition and internal environment maintenance were implemented in accordance with corresponding guidelines.

\section{Clinical and laboratory measurements and recordings}

\section{Scoring systems for disease severity}

APACHEII, LIPS, lung injury score (LIS), Marshall, sequential organ failure assessment (SOFA), and gas exchange, organ failure, cause, associated disease (GOCA) scoring systems were used to assess disease severity upon patients' admission. APACHE II is a disease classification system used to stratify acutely ill patients prognostically by risk of death via 14 basic physiologic principles (22). LIPS consists of 2 major risks: (I) predisposing conditions, including shock, aspiration, sepsis, pneumonia, high-risk surgery, and highrisk trauma; (II) risk modifiers, containing alcohol abuse, body mass index $(\mathrm{BMI})>30 \mathrm{~kg} / \mathrm{m}^{2}$, hypoalbuminemia, chemotherapy, $\mathrm{FiO}_{2}>35 \%$, respiratory rate $>30 \mathrm{bpm}$, $\mathrm{SpO}_{2}<95 \%, \mathrm{pH}<7.35$, and diabetes mellitus (8). SOFA and Marshall scores are organ failure assessment tools developed by consensus in a group of critical care physicians in 1994 and by Marshall through investigating 692 critically ill patients in 1995, respectively, both of which consist of scores from six organ systems and is graded from 0 to 4 according to the degree of dysfunction $(23,24)$. LIS is a score for lung injury released by Murray in 1988, which classify extent of lung injury into 3 categories (25).

\section{Clinical manifestations and laboratory measurements}

At admission, all patients' clinical characteristics including disease histories and durations, symptoms and vital signs, as well as medication histories were recorded. We also recorded the details of treatment for each patient during hospitalization, such as MV settings, type and dose of analgesics and sedatives, and usage of vasopressors and muscle relaxants. Echocardiography and chest computed tomography were performed on the first day of admission, and, at the same time, we also collected the venous and arterial blood to do the blood type measurement, blood cell counting, hepatic, renal and cardiac function test, coagulation analysis, cellular and humoral immunity analysis, and blood gas analysis. For cellular and humoral immunity analysis, we measured the total $\mathrm{T}$ cells and their subtypes including CD4+ helper T cells and CD8+ cytotoxic $\mathrm{T}$ cells, and complement concentrations. Blood, 
sputum, and urine samples were collected upon admission to screen the pathogen by culturing or chemiluminescence.

\section{Statistical analysis}

Data collection and statistical analysis were performed separately by two independent investigators. Continuous variables were reported as mean \pm standard deviation, while dichotomous variables were reported as frequency and proportion. We used SPSS 21.0 [Copyright (c) SPSS Inc. 1989-2007] to test the hypothesis, and statistical significance was rendered as a two-sided $\mathrm{P}$ value of $<0.05$.

Independent-sample Student's $t$-test and Chi-square test or Fisher's exact test were conducted to compare demographic, clinical and prognostic characteristics between patients who developed ARDS and those who did not. Univariate logistic regression with unadjusted odds ratio (OR) and $95 \%$ confidence interval (CI) was performed to identify potential parameters for ARDS development and mortality. Significant predictors with $\mathrm{P}<0.1$ in the univariate logistic regression model were then verified by the multivariate logistic regression to filter the independent risk factors, which were further depicted in receiver operating characteristic (ROC) curves to calculate the area under the curve (AUC) for evaluation of their accuracy in discriminating ARDS from severe pneumonia and death from survival. Cutoff points were identified as Youden index (= sensitivity + specificity -1 ) reached the maximum, and the respective sensitivity and specificity were also calculated. We also performed the correlation matrixes and the variance inflation factors analyses for the variables in the multivariable regression model to evaluate the potential correlations and multicollinearity among individual variables.

\section{Results}

A total of 666 patients in RICU were screened for eligibility, and eventually 157 patients with severe pneumonia were included in our final analysis, of which 43 patients developed ARDS while 114 patients did not, which resulted in a proportion of $27 \%$ of ARDS development in patients with severe pneumonia. The hospital mortality of patients with ARDS development reached 22 (51.2\%); while for patients without ARDS development it was 58 (50.8\%). The study flow was summarized in Figure 1.

\section{Demographic, clinical and microbiological characteristics between severe pneumonia patients with and without ARDS development}

APACHE II, LIPS, and LIS were calculated to be higher while Marshall, SOFA, and GOCA were lower in patients with ARDS development, but significant differences were only found in LIPS, LIS, Marshall and SOFA (Table 1). Patients with ARDS development had less cardiovascular (27.9\% vs. $50.9 \%)$ and gastrointestinal (9.3\% vs. $38.6 \%)$ diseases. However, we did not find any significant differences between the two groups in gender, age, BMI, or other comorbid diseases including diabetes, renal diseases, connective tissue diseases, dermatosis, or metabolic diseases.

Table 2 showed that, compared with patients without ARDS development, disease duration before admission was significantly shorter, proportion of lower extremities edema, $\mathrm{PaO}_{2} / \mathrm{FiO}_{2}, \mathrm{BUN}$ and myoglobin were significantly lower, while body temperature, serum fibrinogen (FiB) and C-reactive protein were significantly higher in patients with ARDS development, but we did not find significant difference in echocardiography, blood types, or cellular and humoral immunity.

A total of 12 species of bacteria, 6 species of fungi, and 13 species of viruses were detected. We did not notice any significant differences in bacteria, fungi, or virus between two groups (Table 3). However, we found that sputum had the highest pathogen detection rate, and bacteria were the predominant microbes in patients with severe pneumonia, of which Acinetobacter Bauman and Candida were the leading bacterium and fungus.

\section{Treatment and outcome characteristics between severe pneumonia patients with and without ARDS development}

In comparison of patients without ARDS development, patients with ARDS development used more invasive MV, higher PEEP or expiratory positive airway pressure, $\mathrm{FiO}_{2}$ during invasive $M V$ and non-invasive $M V$, and dosages of midazolam and use of muscle relaxants (Table 4). However, the need of antibiotics, corticosteroids, diuretics and blood transfusion were similar between the two groups.

During treatment, patients with ARDS development had significantly less delirium than patients without ARDS development, but we did not find significant difference in new-onset septic shock, duration of MV, ICU stay, or mortality (Table 5). 


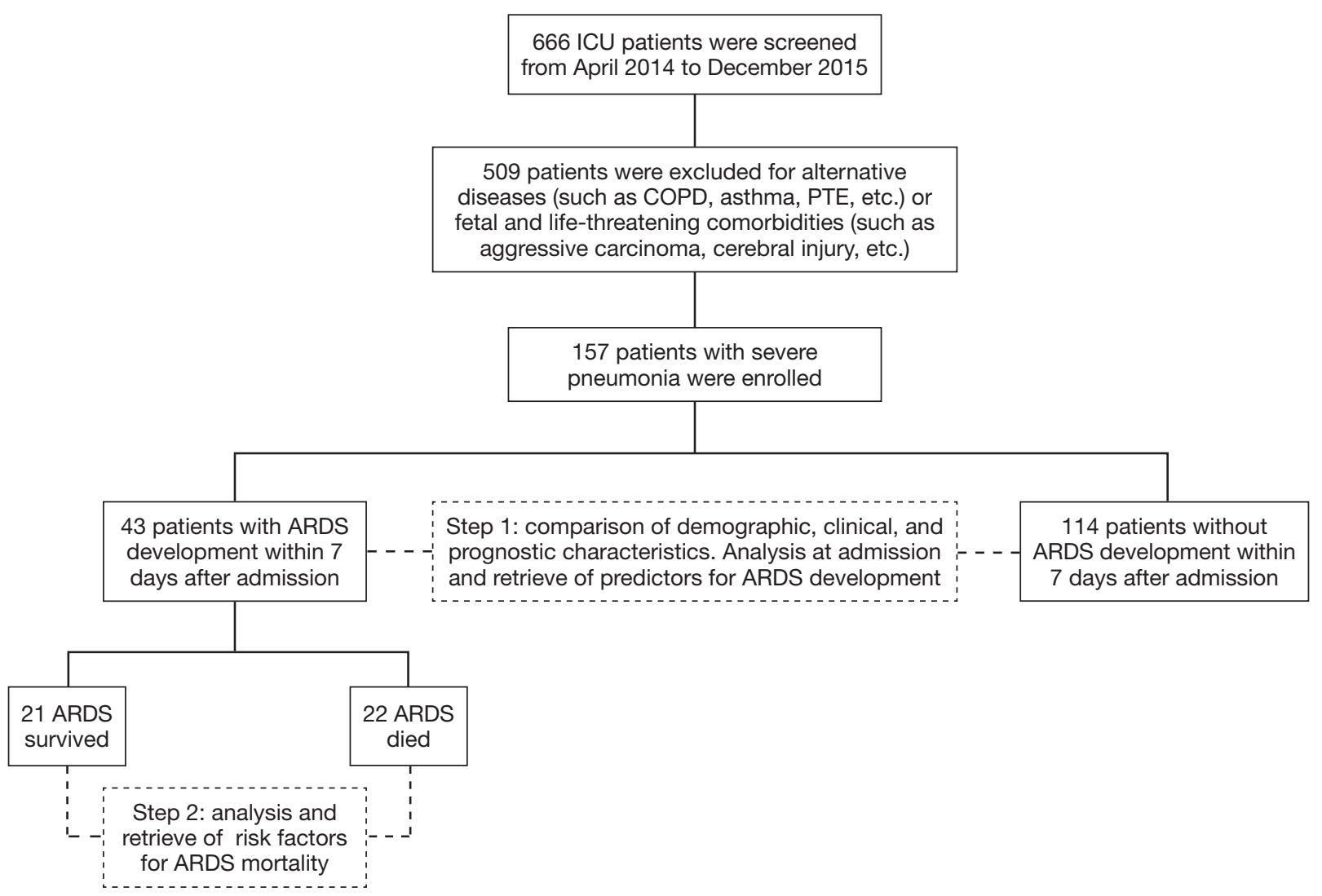

Figure 1 Study flow diagram. ARDS, acute respiratory distress syndrome; COPD, chronic obstructive pulmonary disease; ICU, intensive care unit; PTE, pulmonary thromboembolism.

\section{Predictors and risk factors for ARDS development and prognosis among severe pneumonia}

Tables 6,7 summarized the potential risk factors involving ARDS development and prognosis, respectively, which showed that gastrointestinal diseases, $\mathrm{SpO}_{2}, \mathrm{PaO}_{2} / \mathrm{FiO}_{2}$, and BUN were inversely associated with ARDS development, while LIS score, body temperature, serum FiB, PEEP, $\mathrm{FiO}_{2}$ during invasive $\mathrm{MV}$, and use of muscle relaxants were in positive correlation with ARDS development; and BMI and $\mathrm{pH}$ were in negative correlation with ARDS mortality, while male patients, age, APACHE II score, and serum creatinine were positively associated with ARDS mortality. However, multivariate logistic regression revealed that serum $\mathrm{FiB}$ (adjusted OR 1.893, 95\% CI: $1.141-3.142, \mathrm{P}=0.014$ ) and PEEP (adjusted OR 1.651, 95\% CI: 1.218-2.237, $\mathrm{P}=0.001$ ) were independent risk factors for ARDS development, and $\mathrm{pH}$ (adjusted OR 0.832, 95\% CI: 0.702-0.985, $\mathrm{P}=0.033$ ) was independent risk factor for ARDS mortality. In the correlation matrixes analysis, we found significant correlations between gastroenteric diseases and BUN, body temperature and $\mathrm{PaO}_{2} / \mathrm{FiO}_{2}, \mathrm{PaO}_{2} / \mathrm{FiO}_{2}$ and $\mathrm{BUN}, \mathrm{PEEP}$ and $\mathrm{PaO}_{2} / \mathrm{FiO}_{2}$, and muscle relaxants and gastroenteric diseases, $\mathrm{PaO}_{2} / \mathrm{FiO}_{2}$ and PEEP in multivariate logistic regression model for ARDS development, while for ARDS mortality, gender and creatinine, and $\mathrm{pH}$ and creatinine had potential correlations. (Data not shown) However the variance inflation factor analyses of both outcomes did not show significant multicollinearity (data not shown).

In discrimination of ARDS from severe pneumonia, ROC curves of serum FiB and PEEP resulted in an AUC of 0.661 (95\% CI: $0.525-0.797, \mathrm{P}=0.034)$ and $0.833(95 \%$ CI: $0.733-0.934, \mathrm{P}<0.001)$, respectively, and an cutoff point of $5.15 \mathrm{~g} / \mathrm{L}$ for serum $\mathrm{FiB}$ with a sensitivity and specificity of $58.3 \%$ and $68.4 \%$ and of $6.5 \mathrm{cmH}_{2} \mathrm{O}$ for PEEP with a sensitivity of $87.5 \%$ and a specificity of $65.8 \%$ (Figure 2). Similarly in identification of ARDS mortality, the AUC of $\mathrm{pH}$ was calculated to be 0.778 (95\% CI: 0.641-0.915, $\mathrm{P}=0.002$ ) with a cutoff point of 7.35 yielding a sensitivity and specificity of $95.2 \%$ and $54.5 \%$, respectively (Figure 3). 
Table 1 Baseline characteristics between severe pneumonia patients with and without ARDS development

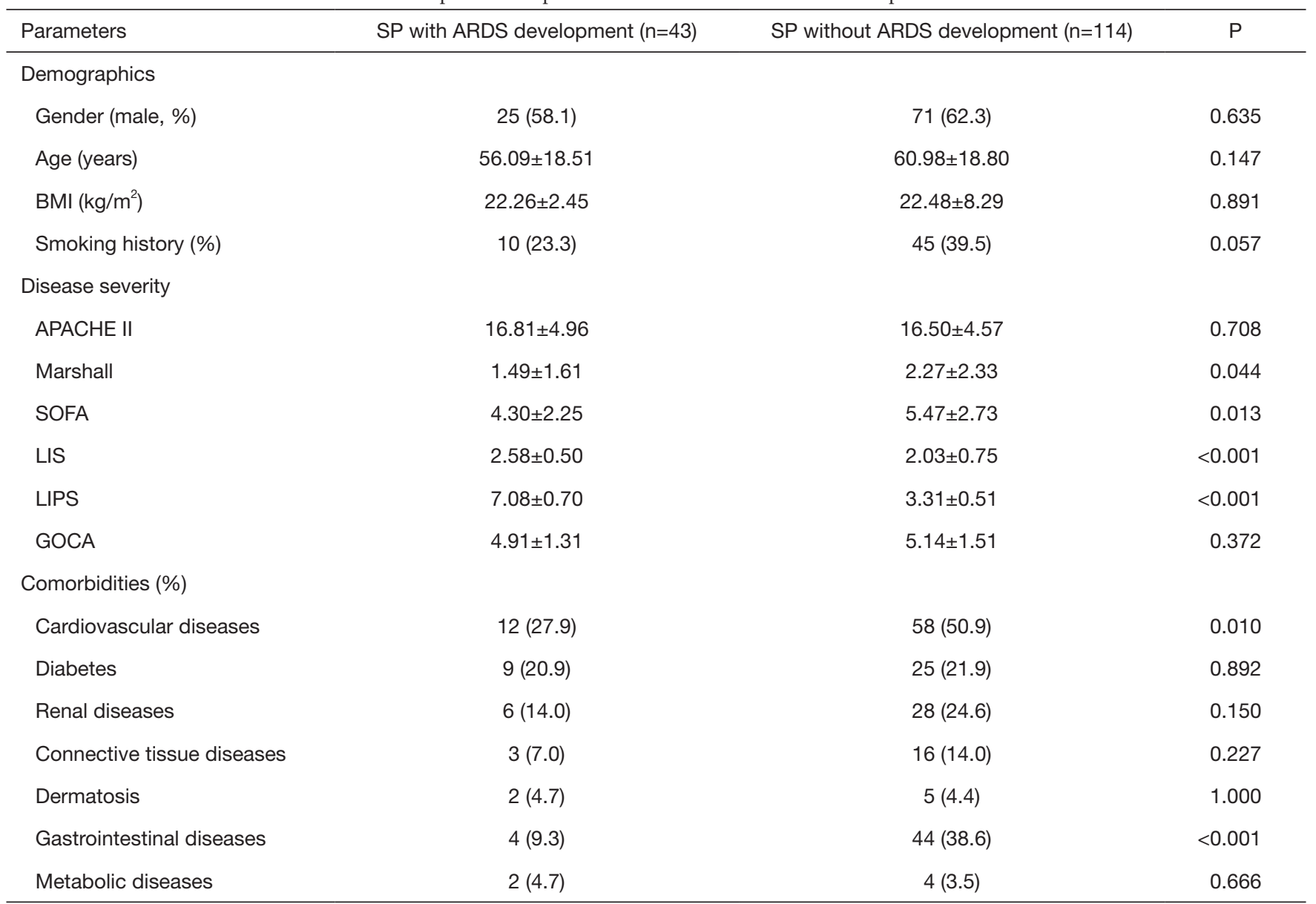

APACHE II, acute physiology, age, chronic health evaluation; ARDS, acute respiratory distress syndrome; BMI, body mass index; GOCA, gas exchange, organ failure, cause, associated disease; LIPS, lung injury prediction score; LIS, lung injury score; SOFA, sequential organ failure assessment; SP, severe pneumonia.

\section{Discussion}

In our study, we found that, in patients who developed ARDS within 7 days after admission, disease duration before admission was shorter, LIS score, body temperature, FiB, C-reactive protein, PEEP, expiratory positive airway pressure, $\mathrm{FiO}_{2}$ during invasive and non-invasive $\mathrm{MV}$, dosages of midazolam, proportion of invasive $\mathrm{MV}$ and muscle relaxants were higher, while Marshall and SOFA score, $\mathrm{SpO}_{2}, \mathrm{PaO}_{2} / \mathrm{FiO}_{2}, \mathrm{BUN}$, myoglobin, cardiovascular and gastrointestinal diseases, and lower extremities edema were lower than those in patients without ARDS development. Serum FiB and PEEP were independent risk factors for ARDS development, and $\mathrm{pH}$ was independent risk factor for ARDS mortality, of which FiB $>5.15 \mathrm{~g} / \mathrm{L}$ and PEEP $>6.5 \mathrm{cmH}_{2} \mathrm{O}$ determined ARDS risk with a power of $58.3 \%$ and $87.5 \%$ in sensitivity and $68.4 \%$ and $65.8 \%$ in specificity, respectively, while $\mathrm{pH}<7.35$ resulted in higher risk of mortality with a sensitivity of $95.2 \%$ and specificity of $54.5 \%$.

Severe pneumonia is a one of the major causes for ICU admission and leads to a high risk of morbidity and mortality even with potent antibiotic therapy and sufficient respiratory support (19,26-28). As described in introduction, ARDS is a severe lung injury with high mortality but lack effective treatment and severe pneumonia is the predominant cause of ARDS, therefore the treatment failure for severe pneumonia might result from ARDS development afterward. It has been reported that many treatments targeting the mechanisms identified in promising preclinical studies fail to improve patient outcomes partially due to the delayed recognition of patients at risk and the 
Table 2 Clinical characteristics and laboratory analysis between severe pneumonia patients with and without ARDS development

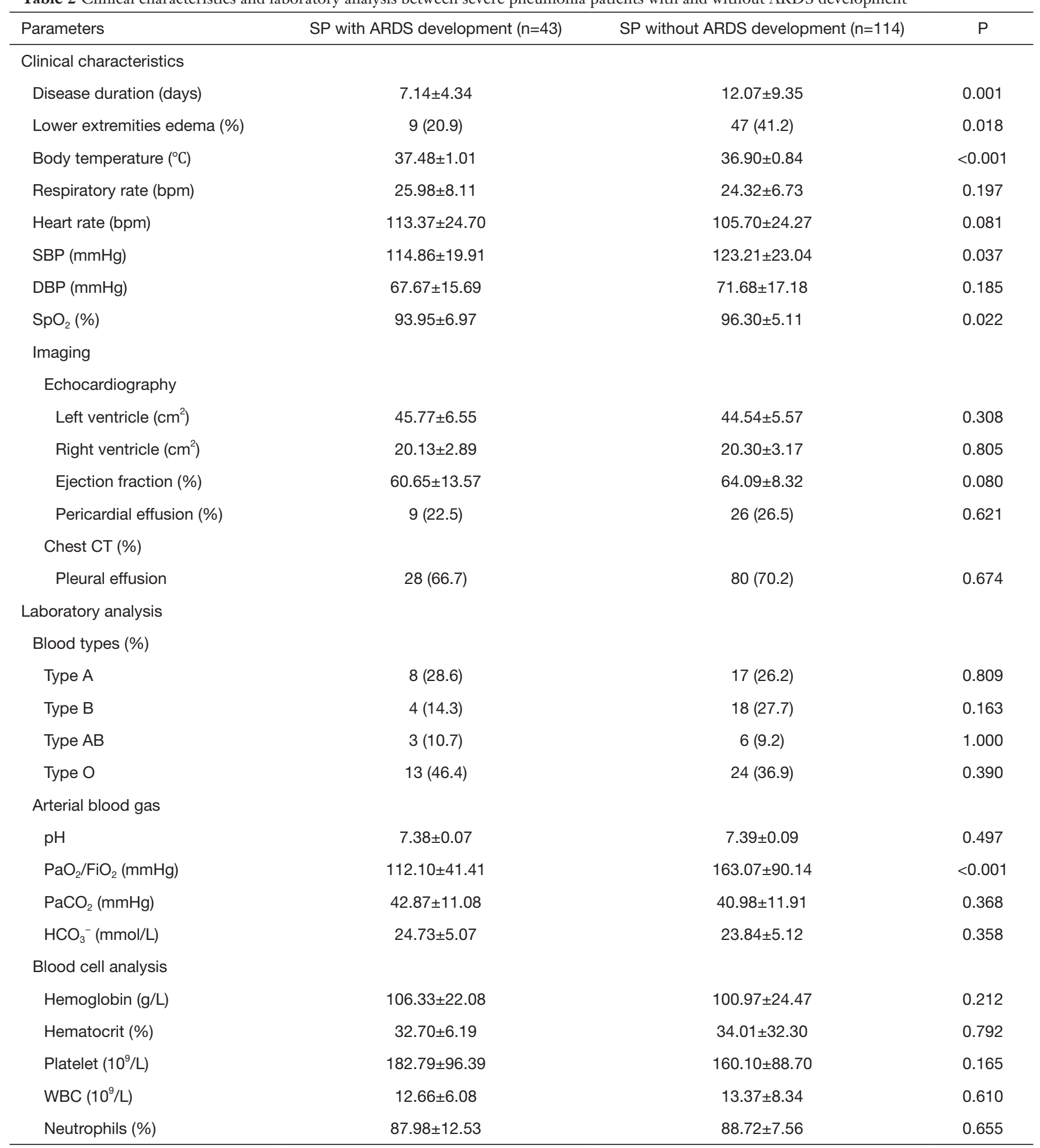

Table 2 (continued) 
Table 2 (continued)

\begin{tabular}{|c|c|c|c|}
\hline Parameters & SP with ARDS development $(n=43)$ & SP without ARDS development $(n=114)$ & $\mathrm{P}$ \\
\hline $\mathrm{TB}(\mu \mathrm{mol} / \mathrm{L})$ & $11.27 \pm 6.15$ & $12.78 \pm 12.62$ & 0.453 \\
\hline ALT (IU/L) & $33.35 \pm 32.92$ & $75.83 \pm 198.65$ & 0.186 \\
\hline AST (IU/L) & $53.51 \pm 35.95$ & $147.06 \pm 581.31$ & 0.294 \\
\hline GGT (IU/L) & $62.26 \pm 52.66$ & $101.04 \pm 234.64$ & 0.285 \\
\hline Albumin (g/L) & $27.61 \pm 4.88$ & $28.03 \pm 4.93$ & 0.627 \\
\hline BUN (mmol/L) & $8.81 \pm 5.52$ & $12.00 \pm 7.92$ & 0.016 \\
\hline Creatinine $(\mu \mathrm{mol} / \mathrm{L})$ & $84.84 \pm 51.73$ & $119.20 \pm 118.81$ & 0.069 \\
\hline PT (s) & $13.66 \pm 2.68$ & $14.54 \pm 3.60$ & 0.155 \\
\hline INR & $1.21 \pm 0.23$ & $1.27 \pm 0.31$ & 0.308 \\
\hline APTT (s) & $40.44 \pm 25.06$ & $39.34 \pm 20.74$ & 0.783 \\
\hline $\mathrm{FiB}(\mathrm{g} / \mathrm{L})$ & $5.15 \pm 2.15$ & $4.20 \pm 1.95$ & 0.010 \\
\hline D-dimer (mg/L FEU) & $231.31 \pm 1,407.25$ & $8.30 \pm 8.40$ & 0.094 \\
\hline \multicolumn{4}{|l|}{ Myocardial biomarkers } \\
\hline Myoglobin (ng/mL) & $139.21 \pm 221.74$ & $383.27 \pm 704.43$ & 0.029 \\
\hline \multicolumn{4}{|l|}{ Inflammatory biomarkers } \\
\hline Interleukin-6 (ng/mL) & $905.87 \pm 1,368.17$ & $452.33 \pm 1,086.52$ & 0.076 \\
\hline \multicolumn{4}{|l|}{ Cellular immunity (\%) } \\
\hline CD3 cell & $60.52 \pm 13.94$ & $56.37 \pm 16.64$ & 0.215 \\
\hline CD4 cell & $27.63 \pm 13.65$ & $28.42 \pm 13.59$ & 0.782 \\
\hline CD8 cell & $29.49 \pm 14.92$ & $24.82 \pm 15.59$ & 0.150 \\
\hline \multicolumn{4}{|l|}{ Humoral immunity $(g / L)$} \\
\hline Complement 3 & $0.8474 \pm 0.2410$ & $0.7647 \pm 0.2569$ & 0.126 \\
\hline Complement 4 & $0.2198 \pm 0.0755$ & $0.1988 \pm 0.0781$ & 0.205 \\
\hline
\end{tabular}

ARDS, acute respiratory distress syndrome; CT, computed tomography; DBP, diastolic blood pressure; $\mathrm{HCO}_{3}{ }^{-}$, bicarbonate ion; $\mathrm{PaCO}_{2}$, partial pressure of arterial carbon dioxide; $\mathrm{PaO}_{2} / \mathrm{FiO}_{2}$, ratio of partial pressure of arterial oxygen to fraction of inspired oxygen; SBP, systolic blood pressure; SP, severe pneumonia; $\mathrm{SpO}_{2}$, oxygen saturation of pulse oximetry; WBC, white blood cell count; ALP, alkaline phosphatase; ALT, alanine aminotransferase; APTT, activated partial thromboplastin time; ARDS, acute respiratory distress syndrome; AST, aspartate aminotransferase; BNP, brain natriuretic peptide; BUN, blood urea nitrogen; CRP, C-reactive protein; FiB, fibrinogen; GGT, gamma-glutamyltranspetidase; INR, international normalized ratio; LDH, lactate dehydrogenase; PT, prothrombin time; SP, severe pneumonia; TB, total bilirubin. 
Table 3 Microbiological analysis between severe pneumonia patients with and without ARDS development

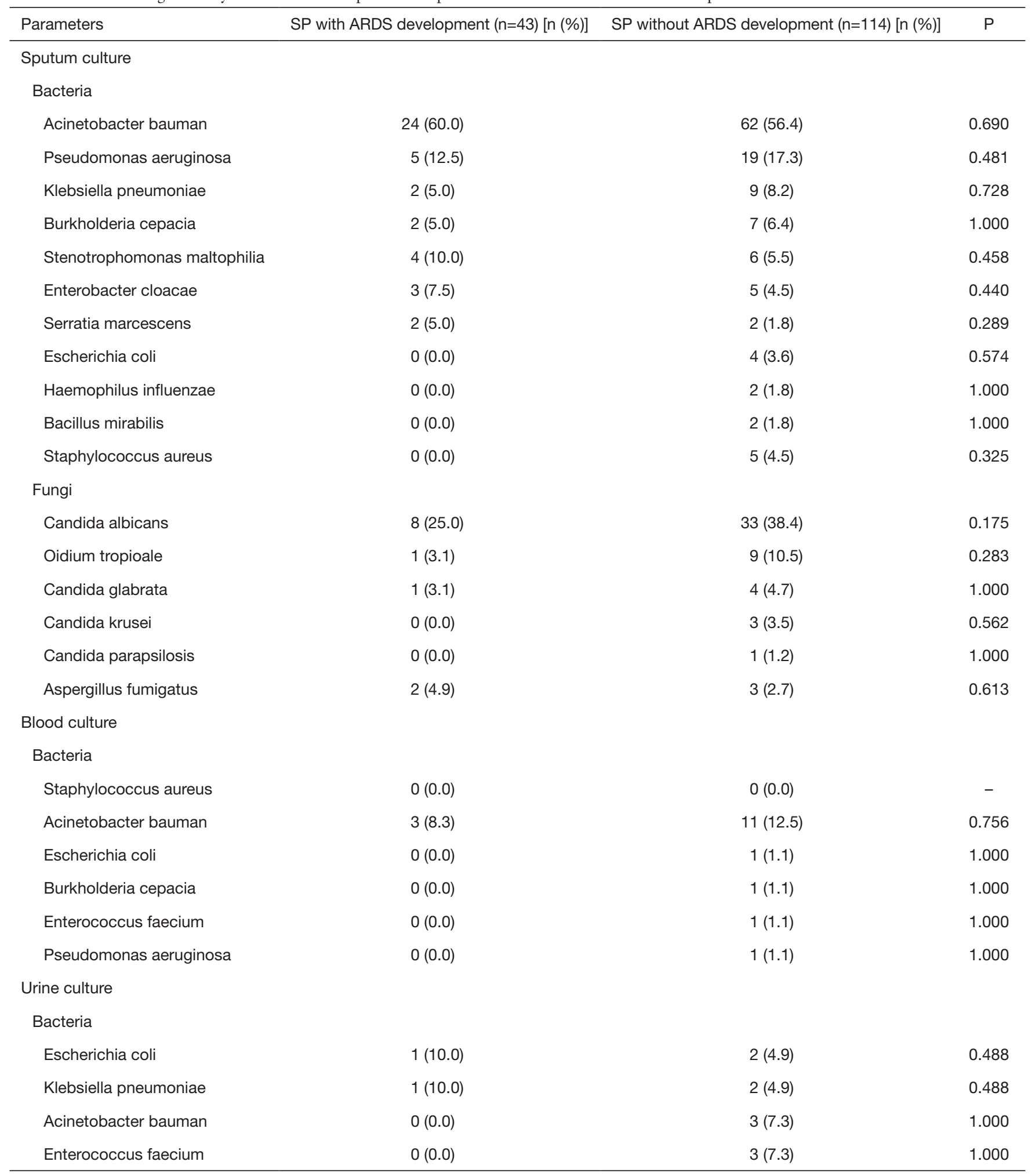

Table 3 (continued) 
Table 3 (continued)

\begin{tabular}{llcc}
\hline Parameters & SP with ARDS development $(n=43)[n(\%)]$ & SP without ARDS development $(n=114)[n(\%)]$ & $P$ \\
\hline Fungi & $0(0.0)$ & $1(2.6)$ & 1.000 \\
Oidium tropioale & $1(11.1)$ & $2(5.1)$ & 0.472 \\
Candida glabrata & $0(0.0)$ & $7(17.9)$ & 0.320 \\
$\quad$ Candida albicans & & & 0.792 \\
Serum viral lgM & $6(17.1)$ & $12(15.2)$ & 1.000 \\
Herpes simplex virus & $1(2.9)$ & $2(2.5)$ & 0.446 \\
Cytomegalovirus & $3(16.7)$ & $7(10.8)$ & \\
Epstein-Barr virus & & &
\end{tabular}

ARDS, acute respiratory distress syndrome; SP, severe pneumonia.

subsequent development of the full-blown syndrome (8), thus early identification of patients at high risk of ARDS development from those with severe pneumonia could potentially help for early intervention and prevent severe pneumonia patients from developing fatal ARDS because these two conditions require different treatment. For high risk of ARDS, preventive strategies may be required such as protective MV optimization (low tidal volume, high PEEP), restrictive transfusion, sepsis management, as well as conservative fluid strategy (29). Furthermore, some novel treatments are now under investigations but they are all based on early recognition of ARDS, such as prehospital use of inhaled steroids, aspirin, or heparin (30-32). Our study about the risk factors for ARDS development in severe pneumonia further enriches and highlights the limited knowledge in such an area and is of clinical importance and practice.

Scoring systems are reliable and convenient tools for clinicians to evaluate disease severity in critically ill patients, of which APACHE II, Marshall, SOFA, and LIS are the most commonly used scores, and we also included LIPS as a novel lung injury prediction system. Our study demonstrated that Marshall and SOFA were significantly lower while LIPS and LIS were dramatically higher in patients with ARDS development than that in patients without ARDS development, but no significant difference was found in APACHE II or GOCA between the two groups. Based on the significant difference in LIPS score between patients with and without ARDS development, we are confident to conclude that the classification of patients and the results in our study are convincing. Inconsistent results in these scoring systems may be attributable to the differences in evaluation of organ systems in each score system. In LIS score, degree of hypoxemia, lung compliance, PEEP and infiltrates in chest X-ray were assessed, while degree of hypoxemia, platelet count, bilirubin, hypotension and creatinine were appraised in Marshall and SOFA score. In our study, we also notified that, in patients with ARDS development, $\mathrm{PaO}_{2} / \mathrm{FiO}_{2}$ was lower and PEEP was higher, which mainly resulted in higher LIS score in patients with ARDS development. At the meantime, the comorbid cardiovascular and gastrointestinal diseases, in which hypotension due to coronary artery disease and hyperbilirubinemia induced by hepatic dysfunction accounted for majority, were lower in patients who developed ARDS, which might offset the effect of lower $\mathrm{PaO}_{2} / \mathrm{FiO}_{2}$ in Marshall and SOFA score. Therefore, APACHE II is more likely to be rendered as a comprehensive assessment tool, while LIS, Marshall and SOFA should be used in priority under certain clinical conditions.

Refractory hypoxemia and decreased lung compliance, resulted from increased permeability in pulmonary vascular as well as increased alveoli trapping and loss of aerated tissue, are clinical hallmarks for ARDS (1). MV is a validated treatment strategy for ARDS, in which low tidal volume of 4 to $6 \mathrm{~mL} / \mathrm{kg}$ combined with high PEEP and $\mathrm{FiO}_{2}$ were termed as "lung protective ventilation" and "open lung approach" and were demonstrated to be effective and safe in improving oxygenation without inducing barotrauma $(20,33)$. In our study, PEEP and $\mathrm{FiO}_{2}$ in patients with ARDS development was significantly higher than those in patients without ARDS development, which was in accordance with the pathophysiological 
Table 4 Treatment strategies between severe pneumonia patients with and without ARDS development

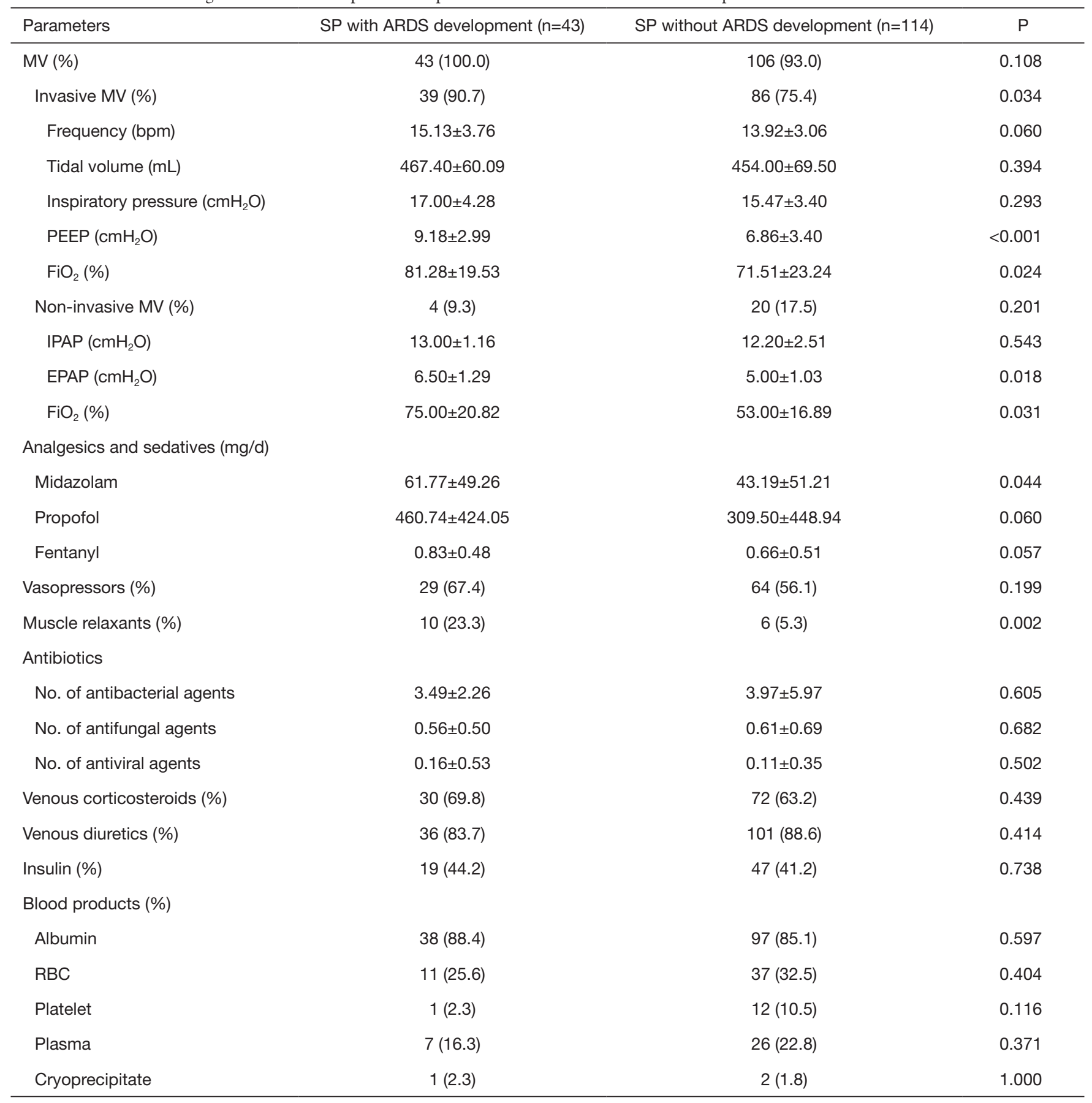

ARDS, acute respiratory distress syndrome; EPAP, expiratory positive airway pressure; $\mathrm{FiO}_{2}$, fraction of inspired oxygen; MV, mechanical ventilation; PEEP, positive end-expiratory pressure; RBC, red blood cell; SP, severe pneumonia.

characteristics of ARDS. However, the level of PEEP in patients who developed ARDS in our study was comparatively lower than that reported by Meade $(9.18 \pm 2.99$ vs. $14.6 \pm 3.4 \mathrm{cmH}_{2} \mathrm{O}$ ) but similar with that reported in our previous study $\left(9.18 \pm 2.99\right.$ vs. $\left.10.3 \pm 3.2 \mathrm{cmH}_{2} \mathrm{O}\right)(3,10)$, and the inconsistence may due to body weight and height as well as races differed in the included patients because higher PEEP may be required in patients with lower 
Table 5 Outcomes measures between severe pneumonia patients with and without ARDS development

\begin{tabular}{lccc}
\hline Parameters & SP with ARDS development $(\mathrm{n}=43)$ & SP without ARDS development $(\mathrm{n}=114)$ & $\mathrm{P}$ \\
\hline Delirium (\%) & $2(4.7)$ & $20(17.5)$ & 0.038 \\
New-onset septic shock (\%) & $9(20.9)$ & $24(21.1)$ & 0.987 \\
Duration of MV (days) & $13.23 \pm 12.88$ & $13.89 \pm 13.85$ & 0.786 \\
Invasive MV (days) & $12.28 \pm 12.99$ & $11.55 \pm 14.06$ & 0.769 \\
Duration of ICU stay (days) & $15.09 \pm 13.66$ & $16.29 \pm 14.75$ & 0.645 \\
Duration of hospital stay (days) & $21.95 \pm 16.96$ & $26.82 \pm 20.47$ & 0.167 \\
Mortality (\%) & $22(51.2)$ & $58(50.9)$ & 0.975 \\
ICU mortality & $12(27.9)$ & $29(25.4)$ & 0.754 \\
Out of ICU mortality & $10(23.3)$ & $29(25.4)$ & 0.778 \\
Cause of death & & & 0.039 \\
Respiratory failure & $14(63.6)$ & $22(37.9)$ & 0.001 \\
MODS & $2(9.1)$ & $28(48.3)$ & 0.386 \\
DIC & $3(13.6)$ & $4(6.9)$ & 0.386 \\
Septic shock & $3(13.6)$ & $4(6.9)$ & \\
\hline
\end{tabular}

ARDS, acute respiratory distress syndrome; DIC, disseminated intravascular coagulation; ICU, intensive care unit; MODS, multiple organ dysfunction syndrome; MV, mechanical ventilation; SP, severe pneumonia.

chest wall compliance induced by overweight. In addition, we also found that, compared with patients without ARDS development, the need of invasive MV, sedatives and muscle relaxants was significantly higher in patients with ARDS development, which further demonstrated the treatment difficulty in ARDS and the urgency and essentiality of early recognition of ARDS.

Since its first report in 1967, ARDS has been defined all the way as an acute onset of bilateral pulmonary infiltration, which induces severe oxygenation dysfunction $(2,34)$. Moreover, recent Berlin definition further limited the timing of ARDS to be less than a week (1). Pneumonia is a form of lung infection, which results in patches in chest imaging $(35,36)$. A wide variety of pathogenic microbes may cause pneumonia, thus the disease duration of pneumonia is solely determined by the pathogen's biological properties. In our study, we found shorter disease duration before admission in patients with ARDS development, which further highlighted the importance of timing for ARDS diagnosis as well as its difference from severe pneumonia. However, to our knowledge, most studies predicting ARDS and investigating prognostic risk factors did not take the timing into consideration, which may lead to heterogeneity in patients as well as inaccuracy in results. Therefore, abiding by the timing defined by Berlin definition, we retrieved and validated potential risk factors to early identify ARDS from patients with severe pneumonia.

In our univariate logistic regression, LIS score, gastrointestinal diseases, muscle relaxants, body temperature, $\mathrm{PaO}_{2} / \mathrm{FiO}_{2}$, BUN, level of serum FiB, and PEEP were further identified to be associated with ARDS development in addition to $\mathrm{SpO}_{2}$ and $\mathrm{FiO}_{2}$ reported in LIPS, but only serum FiB and PEEP were shown to be independently associated with ARDS onset in patients with severe pneumonia in multivariate logistic regression. Moreover, ROC curve demonstrated the high reliability of PEEP in a cutoff point of $6.5 \mathrm{cmH} 2 \mathrm{O}$, and the relatively low reliability of serum FiB in a cutoff point of $5.15 \mathrm{~g} / \mathrm{L}$. In the Berlin definition, a minimum level of PEEP $\left(5 \mathrm{cmH}_{2} \mathrm{O}\right)$ was included in the diagnosis of ARDS with consideration of influences in $\mathrm{PaO}_{2} / \mathrm{FiO}_{2}$. However, that minimum PEEP of $5 \mathrm{cmH}_{2} \mathrm{O}$ was resulted from a consensus of professionals and other requirement of a minimum PEEP levels were not evaluated as they were selected by the panel using face validity criteria to ensure compatibility with prior definitions (1). Therefore, a minimum PEEP of $5 \mathrm{cmH}_{2} \mathrm{O}$ was not suitable for any clinical conditions and different minimum levels of PEEP may be demanded in certain disease 
Table 6 Univariate and multivariate logistic regression analysis of risk factors for ARDS development in severe pneumonia

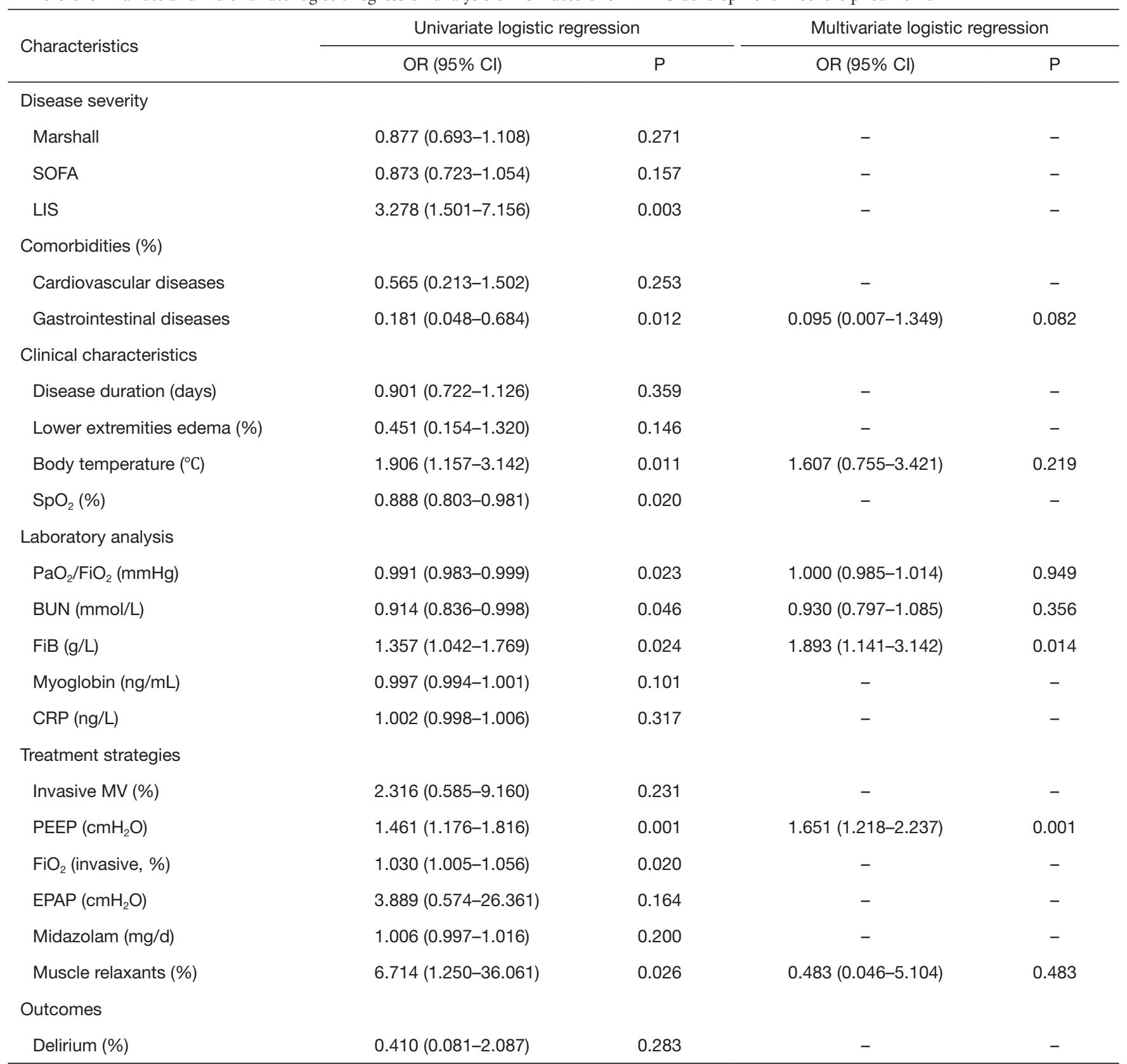

ARDS, acute respiratory distress syndrome; BUN, blood urea nitrogen; Cl, confidence interval; CRP, C-reactive protein; EPAP, expiratory positive airway pressure; $\mathrm{FiB}$, fibrinogen; $\mathrm{FiO}_{2}$, fraction of inspired oxygen; LIS, lung injury score; MV, mechanical ventilation; PEEP, positive end-expiratory pressure; OR, odds ratio; $\mathrm{PaO}_{2} / \mathrm{FiO}$, ratio of partial pressure of arterial oxygen to fraction of inspired oxygen; SOFA, sequential organ failure assessment; $\mathrm{SpO}_{2}$, oxygen saturation of pulse oximetry.

circumstances, just as a higher PEEP of $6.5 \mathrm{cmH}_{2} \mathrm{O}$ reported in our study. As for the discrimination capacity of serum level of FiB, several studies have demonstrated that coagulation and fibrinolytic pathways play an active role in modulating systemic inflammatory processes in patients with severe sepsis and the severity of abnormalities in theses pathways correlates with clinical outcomes, of which plasminogen activator inhibitor-1, impairing fibrinolysis by the inhibition of tissue plasminogen activator and urokinase and resulting in accumulation of $\mathrm{FiB}$, was found to be an independent risk factor for mortality in 
Table 7 Univariate and multivariate logistic regression analysis of risk factors for ARDS mortality in severe pneumonia

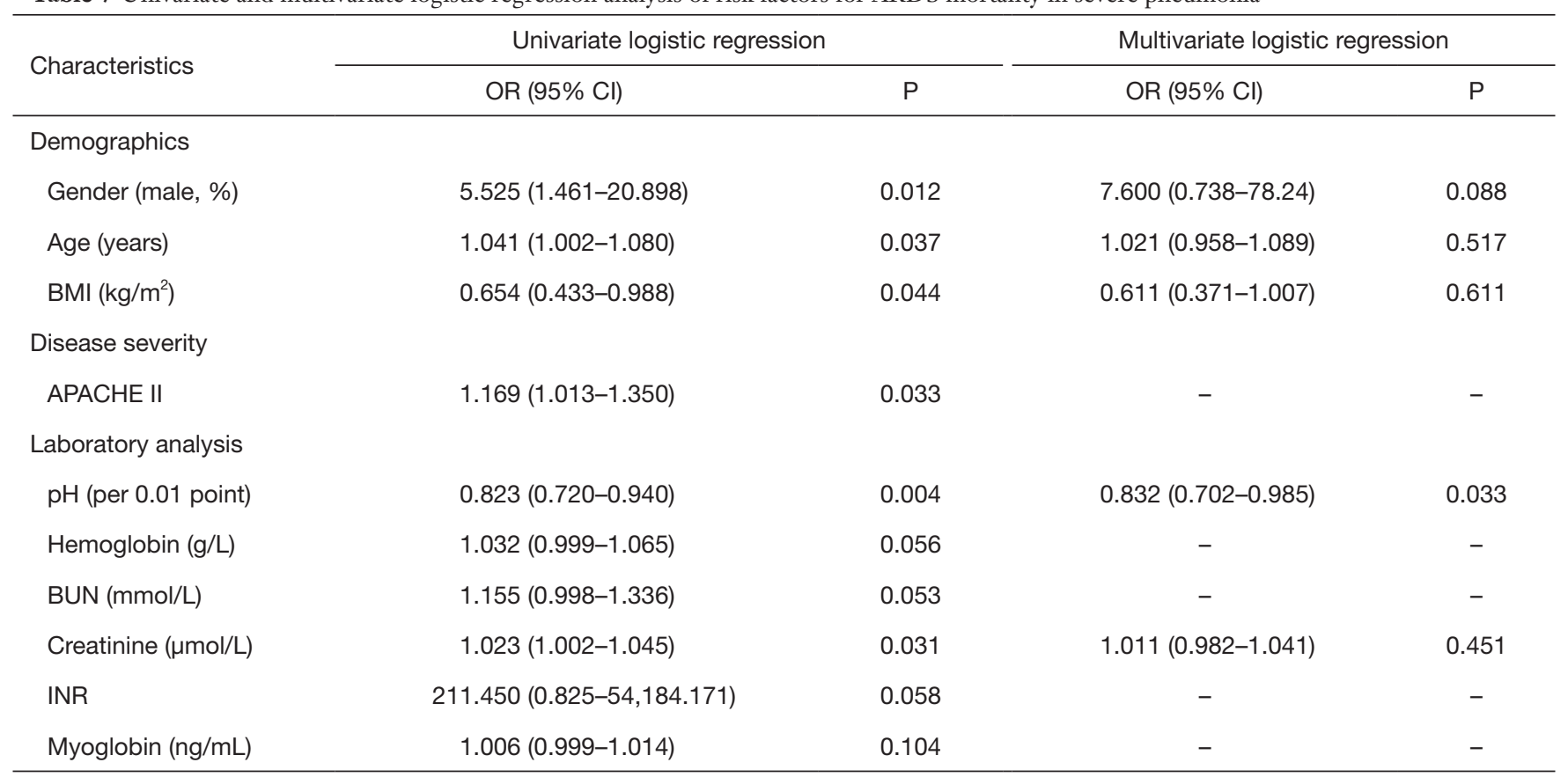

APACHE II, acute physiology, age, chronic health evaluation; ARDS, acute respiratory distress syndrome; BMI, body mass index; BUN, blood urea nitrogen; $\mathrm{Cl}$, confidence interval; INR, international normalized ratio; OR, odds ratio.

patients with ARDS (37-39).

A large number of prognostic factors have been verified in previous studies, including factors in clinical characteristics, physiological parameters, genetic polymorphisms, biomarkers and scoring systems (15). In our study, we found that higher mortality in patients with ARDS was associated with older age, higher APACHE II score, creatinine level and use of vasopressors and new-onset septic shock, but lower $\mathrm{BMI}$ and $\mathrm{pH}$, which were in accordance with the recent findings $(40,41)$. However, multivariate logistic regression analysis showed that all these parameters were confounding factors except for $\mathrm{pH}$, which had a high sensitivity of $95.2 \%$ in a cutoff point of 7.35 . The value of $\mathrm{pH}$ in our study was higher than that reported in the studies by Brun-Buisson and Villar (7.35 vs. 7.30), and the difference may due to the inconsistence in patients selection $(42,43)$. Controversies lies in the association between $\mathrm{PaO}_{2} / \mathrm{FiO}_{2}$ at the onset of ARDS and the adverse outcomes in ARDS (42-45), but our study revealed that initial $\mathrm{PaO}_{2} / \mathrm{FiO}_{2}$ is not an independent predictor of mortality, which we suspected was attributed to the insignificant difference in PEEP because $\mathrm{PaO}_{2} / \mathrm{FiO}_{2}$ has been reported to vary depending on ventilator strategy and is sensitive to ventilator settings (46), and early change of $\mathrm{PaO}_{2} / \mathrm{FiO}_{2}$ being too likely to be associated with outcomes in ARDS (10,47).

Limitations for our study included: Firstly, relatively small sample size, which may lead to insignificant differences of some potential predictors in statistical analysis; Secondly, inadequate parameters recorded such as but not limited to alcohol abuse condition, respiratory mechanisms including inspiratory plateau pressure and lung compliance, early alteration of $\mathrm{PaO}_{2} / \mathrm{FiO}_{2}$, as well as some candidate genes; Thirdly, $\mathrm{FiO}_{2}$ was excluded in our study due to the correlation with $\mathrm{PaO}_{2} / \mathrm{FiO}_{2}$, which might omit this potential risk factor for ARDS because $\mathrm{PaO}_{2} / \mathrm{FiO}_{2}$ depended on $\mathrm{FiO}_{2}$ and $\mathrm{FiO}_{2}$ changes would change the disease classification (48); Finally, the potential overlap and correlation between individual risk factors, such as gastroenteric diseases and urea nitrogen level, body temperature and $\mathrm{PaO}_{2} / \mathrm{FiO}_{2}$, and $\mathrm{PaO}_{2} / \mathrm{FiO}_{2}$ and PEEP in the correlation matrix analysis (data not shown), may offset and cover the role of some risk factors in the multivariate logistic regression.

\section{Conclusions}

Patients with ARDS development have different clinical characteristics and need distinct treatment strategies 


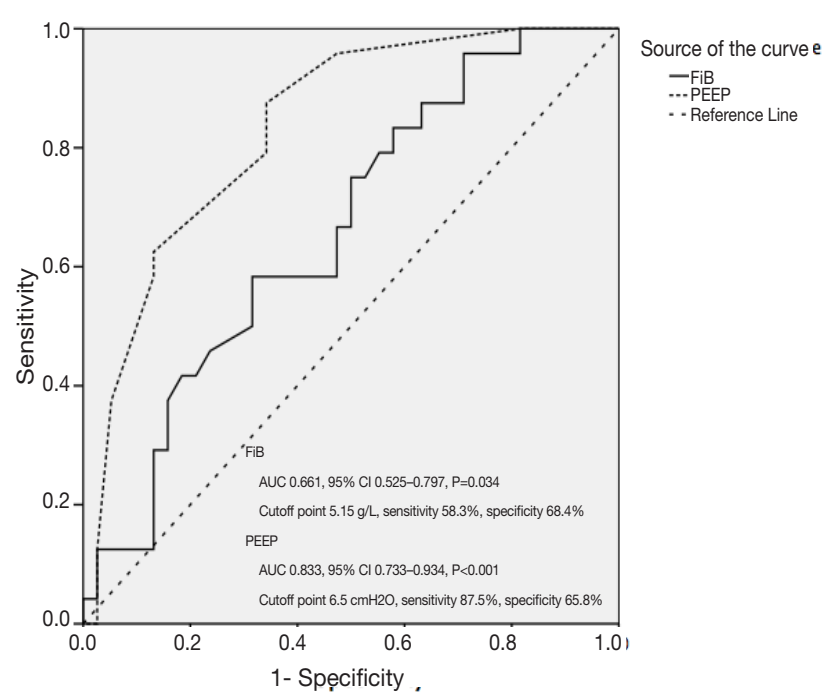

Figure 2 ROC curves of FiB and PEEP in identifying risks for ARDS development in severe pneumonia. ROC curves show accuracy and power of FiB and PEEP in distinguishing patients at risk of ARDS from patients with severe pneumonia. The solid line represented FiB, while the dotted line represented PEEP. The AUC of FiB and PEEP was 0.661 (95\% CI: 0.525-0.797, P=0.034) and 0.833 (95\% CI: $0.733-0.934, \mathrm{P}<0.001$ ), and the cutoff point was calculated to be $5.15 \mathrm{~g} / \mathrm{L}$ and $6.5 \mathrm{cmH}_{2} \mathrm{O}$ with a sensitivity of $58.3 \%$ and $87.5 \%$ and a specificity of $68.4 \%$ and $65.8 \%$, respectively. ARDS, acute respiratory distress syndrome; AUC, area under the curve; CI, confidence interval; FiB, fibrinogen; PEEP, positive endexpiratory pressure; ROC, receiver operating characteristic.

compared to patients with severe pneumonia. A minimum of PEEP $>6.5 \mathrm{cmH}_{2} \mathrm{O}$ and level of serum $\mathrm{FiB}>5.15 \mathrm{~g} / \mathrm{L}$ could sensitively distinguish patients at high risk of ARDS from severe pneumonia, and $\mathrm{pH}<7.35$ is a reliable prognostic factor in predicting risk of mortality in patients with ARDS. More future studies are warranted to further validate and explore potential predictors and possible mechanisms to improve treatment efficacy and create novel treatment strategies for patients with ARDS.

\section{Acknowledgements}

We thank Professor Dongtao Lin (College of Foreign Languages, Sichuan University), who is specialized in biomedical writing and editing, for copyediting this manuscript.

Funding: This work was supported by the Ministry of Science and Technology of the People's Republic of China [2015BAI12B10].

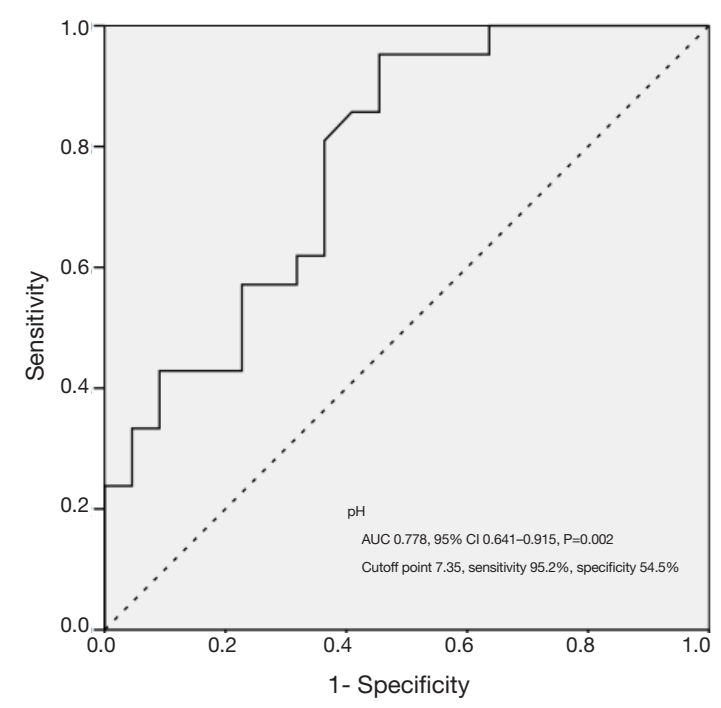

Figure 3 ROC curve of $\mathrm{pH}$ in identifying risks for ARDS mortality in severe pneumonia. ROC curve presents accuracy and power of $\mathrm{pH}$ in predicting risk of mortality for patients with ARDS. The solid line represented $\mathrm{pH}$, and the AUC was 0.778 (95\% CI: $0.641-0.915, \mathrm{P}=0.002$ ) with a sensitivity of $95.2 \%$ and a specificity of $54.5 \%$ at a cutoff point of 7.35 . ARDS, acute respiratory distress syndrome; AUC, area under the curve; CI, confidence interval; ROC, receiver operating characteristic.

\section{Footnote}

Conflicts of Interest: The authors have no conflicts of interest to declare.

Ethical Statement: The study protocol was approved by the Institutional Ethical Committee for Clinical and Biomedical Research of West China Hospital (No. ChiCTRTRC-14004163; Universal Trial Number (UTN): U11111152-2390) and written informed consent was obtained from all patients.

\section{References}

1. ARDS Definition Task Force, Ranieri VM, Rubenfeld GD, et al. Acute respiratory distress syndrome: the Berlin Definition. JAMA 2012;307:2526-33.

2. Bernard GR, Artigas A, Brigham KL, et al. The AmericanEuropean Consensus Conference on ARDS. Definitions, mechanisms, relevant outcomes, and clinical trial coordination. Am J Respir Crit Care Med 1994;149:818-24.

3. Meade MO, Cook DJ, Guyatt GH, et al. Ventilation strategy using low tidal volumes, recruitment maneuvers, 
and high positive end-expiratory pressure for acute lung injury and acute respiratory distress syndrome: a randomized controlled trial. JAMA 2008;299:637-45.

4. Ferguson ND, Cook DJ, Guyatt GH, et al. Highfrequency oscillation in early acute respiratory distress syndrome. N Engl J Med 2013;368:795-805.

5. Bellani G, Laffey JG, Pham T, et al. Epidemiology, Patterns of Care, and Mortality for Patients With Acute Respiratory Distress Syndrome in Intensive Care Units in 50 Countries. JAMA 2016;315:788-800.

6. Jepsen S, Herlevsen P, Knudsen P, et al. Antioxidant treatment with $\mathrm{N}$-acetylcysteine during adult respiratory distress syndrome: a prospective, randomized, placebocontrolled study. Crit Care Med 1992;20:918-23.

7. Zeiher BG, Artigas A, Vincent JL, et al. Neutrophil elastase inhibition in acute lung injury: results of the STRIVE study. Crit Care Med 2004;32:1695-702.

8. Gajic O, Dabbagh O, Park PK, et al. Early identification of patients at risk of acute lung injury: evaluation of lung injury prediction score in a multicenter cohort study. Am J Respir Crit Care Med 2011;183:462-70.

9. Litell JM, Gong MN, Talmor D, et al. Acute lung injury: prevention may be the best medicine. Respir Care 2011;56:1546-54.

10. Luo J, Wang MY, Liang BM, et al. Initial synchronized intermittent mandatory ventilation versus assist/control ventilation in treatment of moderate acute respiratory distress syndrome: a prospective randomized controlled trial. J Thorac Dis 2015;7:2262-73.

11. Rubenfeld GD, Caldwell E, Peabody E, et al. Incidence and outcomes of acute lung injury. N Engl J Med 2005;353:1685-93.

12. Gibelin A, Parrot A, Maitre B, et al. Acute respiratory distress syndrome mimickers lacking common risk factors of the Berlin definition. Intensive Care Med 2016;42:164-72.

13. Mekontso Dessap A, Boissier F, Charron C, et al. Acute cor pulmonale during protective ventilation for acute respiratory distress syndrome: prevalence, predictors, and clinical impact. Intensive Care Med 2016;42:862-70.

14. Ong DS, Klein Klouwenberg PM, Verduyn Lunel FM, et al. Cytomegalovirus seroprevalence as a risk factor for poor outcome in acute respiratory distress syndrome. Crit Care Med 2015;43:394-400.

15. Chen W, Ware LB. Prognostic factors in the acute respiratory distress syndrome. Clin Transl Med 2015;4:65.

16. Trillo-Alvarez C, Cartin-Ceba R, Kor DJ, et al. Acute lung injury prediction score: derivation and validation in a population-based sample. Eur Respir J 2011;37:604-9.
17. Kim JB, Lee SW, Park SI, et al. Risk factor analysis for postoperative acute respiratory distress syndrome and early mortality after pneumonectomy: the predictive value of preoperative lung perfusion distribution. J Thorac Cardiovasc Surg 2010;140:26-31.

18. Chen SW, Chang $\mathrm{CH}$, Chu $\mathrm{PH}$, et al. Risk factor analysis of postoperative acute respiratory distress syndrome in valvular heart surgery. J Crit Care 2016;31:139-43.

19. Mandell LA, Wunderink RG, Anzueto A, et al. Infectious Diseases Society of America/American Thoracic Society consensus guidelines on the management of communityacquired pneumonia in adults. Clin Infect Dis 2007;44 Suppl 2:S27-72.

20. Acute Respiratory Distress Syndrome Network, Brower RG, Matthay MA, et al. Ventilation with lower tidal volume as compared with traditional tidal volumes for acute lung injury and the acute respiratory distress syndrome. N Engl J Med 2000;342:1301-8.

21. Patel SB, Kress JP. Sedation and analgesia in the mechanically ventilated patient. Am J Respir Crit Care Med 2012;185:486-97.

22. Knaus WA, Draper EA, Wagner DP, et al. APACHE II: a severity of disease classification system. Crit Care Med 1985;13:818-29.

23. Vincent JL, Moreno R, Takala J, et al. The SOFA (Sepsisrelated Organ Failure Assessment) score to describe organ dysfunction/failure. On behalf of the Working Group on Sepsis-Related Problems of the European Society of Intensive Care Medicine. Intensive Care Med 1996;22:707-10.

24. Marshall JC, Cook DJ, Christou NV, et al. Multiple organ dysfunction score: a reliable descriptor of a complex clinical outcome. Crit Care Med 1995;23:1638-52.

25. Murray JF, Matthay MA, Luce JM, et al. An expanded definition of the adult respiratory distress syndrome. Am Rev Respir Dis 1988;138:720-3.

26. Restrepo MI, Anzueto A. Severe community-acquired pneumonia. Infect Dis Clin North Am 2009;23:503-20.

27. Rosón B, Carratalà J, Dorca J, et al. Etiology, reasons for hospitalization, risk classes, and outcomes of communityacquired pneumonia in patients hospitalized on the basis of conventional admission criteria. Clin Infect Dis 2001;33:158-65.

28. Carrillo A, Gonzalez-Diaz G, Ferrer M, et al. Noninvasive ventilation in community-acquired pneumonia and severe acute respiratory failure. Intensive Care Med 2012;38:458-66.

29. de Haro C, Martin-Loeches I, Torrents E, et al. Acute 
respiratory distress syndrome: prevention and early recognition. Ann Intensive Care 2013;3:11.

30. Festic E, Ortiz-Diaz E, Lee A, et al. Prehospital use of inhaled steroids and incidence of acute lung injury among patients at risk. J Crit Care 2013;28:985-91.

31. Chen W, Janz DR, Bastarache JA, et al. Prehospital aspirin use is associated with reduced risk of acute respiratory distress syndrome in critically ill patients: a propensityadjusted analysis. Crit Care Med 2015;43:801-7.

32. Festic E, Kor DJ, Gajic O. Prevention of acute respiratory distress syndrome. Curr Opin Crit Care 2015;21:82-90.

33. Amato MB, Barbas CS, Medeiros DM, et al. Beneficial effects of the "open lung approach" with low distending pressures in acute respiratory distress syndrome. A prospective randomized study on mechanical ventilation. Am J Respir Crit Care Med 1995;152:1835-46.

34. Ashbaugh DG, Bigelow DB, Petty TL, et al. Acute respiratory distress in adults. Lancet 1967;2:319-23.

35. Niederman MS, Bass JB Jr, Campbell GD, et al. Guidelines for the initial management of adults with community-acquired pneumonia: diagnosis, assessment of severity, and initial antimicrobial therapy. American Thoracic Society. Medical Section of the American Lung Association. Am Rev Respir Dis 1993;148:1418-26.

36. Bartlett JG, Breiman RF, Mandell LA, et al. Communityacquired pneumonia in adults: guidelines for management. The Infectious Diseases Society of America. Clin Infect Dis 1998;26:811-38.

37. Lorente JA, García-Frade LJ, Landín L, et al. Time course of hemostatic abnormalities in sepsis and its relation to outcome. Chest 1993;103:1536-42.

38. Kinasewitz GT, Yan SB, Basson B, et al. Universal changes in biomarkers of coagulation and inflammation occur in patients with severe sepsis, regardless of causative microorganism. Crit Care 2004;8:R82-90.

39. Ware LB, Matthay MA, Parsons PE, et al. Pathogenetic and prognostic significance of altered coagulation and fibrinolysis in acute lung injury/acute respiratory distress syndrome. Crit Care Med 2007;35:1821-8.

Cite this article as: Luo $\mathrm{J}, \mathrm{Yu} \mathrm{H}, \mathrm{Hu} \mathrm{YH}$, Liu D, Wang YW, Wang MY, Liang BM, Liang ZA. Early identification of patients at risk for acute respiratory distress syndrome among severe pneumonia: a retrospective cohort study. J Thorac Dis 2017;9(10):3979-3995. doi:10.21037/jtd.2017.09.20
40. Monchi M, Bellenfant F, Cariou A, et al. Early predictive factors of survival in the acute respiratory distress syndrome. A multivariate analysis. Am J Respir Crit Care Med 1998;158:1076-81.

41. Luhr OR, Antonsen K, Karlsson M, et al. Incidence and mortality after acute respiratory failure and acute respiratory distress syndrome in Sweden, Denmark, and Iceland. The ARF Study Group. Am J Respir Crit Care Med 1999;159:1849-61.

42. Brun-Buisson C, Minelli C, Bertolini G, et al. Epidemiology and outcome of acute lung injury in European intensive care units. Results from the ALIVE study. Intensive Care Med 2004;30:51-61.

43. Villar J, Ambrós A, Soler JA, et al. Age, PaO2/FIO2, and Plateau Pressure Score: A Proposal for a Simple Outcome Score in Patients With the Acute Respiratory Distress Syndrome. Crit Care Med 2016;44:1361-9.

44. Luhr OR, Karlsson M, Thorsteinsson A, et al. The impact of respiratory variables on mortality in non-ARDS and ARDS patients requiring mechanical ventilation. Intensive Care Med 2000;26:508-17.

45. Bersten AD, Edibam C, Hunt T, et al. Incidence and mortality of acute lung injury and the acute respiratory distress syndrome in three Australian States. Am J Respir Crit Care Med 2002;165:443-8.

46. Villar J, Pérez-Méndez L, Blanco J, et al. A universal definition of ARDS: the $\mathrm{PaO} 2 / \mathrm{FiO} 2$ ratio under a standard ventilatory setting--a prospective, multicenter validation study. Intensive Care Med 2013;39:583-92.

47. Bone RC, Maunder R, Slotman G, et al. An early test of survival in patients with the adult respiratory distress syndrome. The $\mathrm{PaO} 2 / \mathrm{FIo} 2$ ratio and its differential response to conventional therapy. Prostaglandin E1 Study Group. Chest 1989;96:849-51.

48. Karbing DS, Kjaergaard S, Smith BW, et al. Variation in the $\mathrm{PaO} 2 / \mathrm{FiO} 2$ ratio with $\mathrm{FiO} 2$ : mathematical and experimental description, and clinical relevance. Crit Care 2007;11:R118. 